\title{
A characterization of the single-peaked single-crossing domain
}

\section{Edith Elkind ${ }^{1} \cdot$ Piotr Faliszewski ${ }^{2} \cdot$ Piotr Skowron $^{3}$}

Received: 24 August 2018 / Accepted: 3 September 2019 / Published online: 9 September 2019

(c) The Author(s) 2019

\begin{abstract}
We characterize elections that are simultaneously single-peaked and single-crossing (SPSC), by establishing a connection between this domain and that of minimally rich elections, i.e., elections where each candidate is ranked first by at least one voter. Specifically, we show that an election is both single-peaked and single-crossing if and only if it can be obtained from a minimally rich single-crossing election by deleting voters.
\end{abstract}

\section{Introduction}

Perhaps the most famous result in social choice is Arrow's impossibility theorem (Arrow 1951), which establishes that there is no perfect method of aggregating voters' preferences over three or more candidates into a collective opinion. However, this impossibility result only applies if there are no constraints on how the voters may rank the candidates. Thus, a common strategy to circumvent Arrow's theorem is to consider restricted preference domains, i.e., to assume that voters' preferences have additional structure. Under such assumptions, one can often develop aggregation procedures that have a number of desirable properties.

A preliminary version of this paper was presented at the 28th Conference on Artificial Intelligence (AAAI-2014).

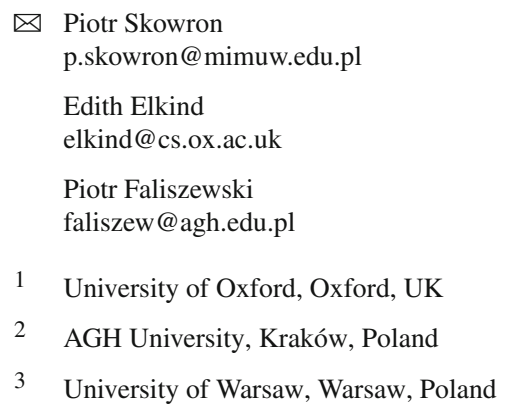


A prominent example of a restricted domain is that of single-peaked preferences (Black 1948). Informally, in single-peaked elections all candidates can be ordered along a single axis, each voter has a most preferred point on this axis, and each voter ranks all candidates so that if candidate $a$ lies between candidate $b$ and the voter's most preferred point then this voter prefers $a$ to $b$ (see Sect. 2). This restricted domain has a number of useful properties: every single-peaked election has a weak Condorcet winner (i.e., a candidate preferred to every other candidate by a weak majority of the voters), its majority relation is transitive (i.e., if a majority of the voters prefer $a$ to $b$ and a majority of the voters prefer $b$ to $c$, then a majority of the voters prefer $a$ to $c$ ) (Inada 1969), and single-peaked elections admit a strategyproof voting rule (Moulin 1980). It was recently established that such elections can be characterized in terms of forbidden configurations (Ballester and Haeringer 2011): there are two elections (one containing two voters and four candidates, and the other containing three voters and three candidates) such that an election is single-peaked if and only if it does not contain subelections that are equivalent to one of these two elections.

Another well-studied restricted domain is that of single-crossing elections (Mirrlees 1971; Roberts 1977). In such elections, the voters can be ordered so that for every pair of candidates their "trajectories" in the voters' preferences intersect at most once, i.e., for every pair of candidates $a, b$ it holds that if the first voter in the ordering ranks $a$ above $b$ then the voters who prefer $a$ to $b$ form a prefix of the ordering. Single-crossing preferences play an important role in the analysis of income redistribution (Mirrlees 1971; Roberts 1977; Meltzer and Richard 1981), coalition formation (Demange 1994), and strategic voting (Saporiti and Tohmé 2006; Saporiti 2009; Barberà and Moreno 2011). Single-crossing elections share some of the desirable properties of singlepeaked elections: for instance, every single-crossing election has a weak Condorcet winner, and Bredereck et al. (2013) show that single-crossing elections, too, can be characterized in terms of a small number of forbidden configurations. However, neither of the restrictions implies the other.

Computational complexity considerations provide another reason to be interested in restricted preferences: single-peaked and single-crossing elections often admit efficient algorithms for social choice problems that are hard for elections with unrestricted preferences. This observation has recently led to a new wave of interest in restricted domains within the computational social choice community (Walsh 2007; Conitzer 2009; Faliszewski et al. 2011b, a; Cornaz et al. 2012, 2013; Betzler et al. 2013; Skowron et al. 2015; Brandt et al. 2015; Dey and Misra 2016; Misra et al. 2017; Jaeckle et al. 2018; Lakhani et al. 2019); see also the survey by Elkind et al. (2017).

Thus, the existing body of work provides us with a good understanding of the properties of elections that are single-peaked or single-crossing. Against this background, the goal of this paper is to characterize the elections that belong to the intersection of these two domains; we refer to the resulting class of elections as the SPSC domain. One of the reasons to be interested in the SPSC domain is the fact that it contains a rich and natural class of elections, namely, the 1-Euclidean elections. These are elections where both voters and candidates can be identified with points on the real line so that each voter prefers the candidates who are closer to her to ones that are further away. The observation that 1-Euclidean elections are both single-peaked and single-crossing dates back to Grandmont (1978). On the other hand, it is known that there exist SPSC 
elections that are not 1-Euclidean, i.e., the SPSC domain is strictly larger than the 1Euclidean domain; the first such example can be found in the work of Coombs (1950), and this also follows from the fact that 1-Euclidean elections cannot be characterized by finitely many forbidden configurations (Chen et al. 2017). While the 1-Euclidean domain admits a very intuitive geometric description, it is more difficult to work with analytically than the single-peaked or the single-crossing domain: for instance, while the latter two domains admit purely combinatorial recognition algorithms (Bartholdi and Trick 1986; Doignon and Falmagne 1994; Escoffier et al. 2008; Elkind et al. 2012; Bredereck et al. 2013), all known algorithms for recognizing 1-Euclidean elections are based on solving linear programs (Doignon and Falmagne 1994; Knoblauch 2010). ${ }^{1}$ Thus, informally, the SPSC domain can be viewed as a combinatorial approximation of the 1-Euclidean domain.

We have mentioned that both the single-peaked domain and the single-crossing domain can be characterized in terms of forbidden configurations; consequently, this is also true for the SPSC domain. However, the resulting description is not particularly intuitive. In this paper, we offer an alternative characterization of the SPSC domain. A notion that turns out to be useful in this context is that of minimal richness: an election is minimally rich if every candidate is ranked first in at least one vote. We show that an election is both single-peaked and single-crossing if and only if it can be obtained from a minimally rich single-crossing election by deleting voters. We develop two combinatorial algorithms that, given an SPSC election, identify a minimally rich single-crossing election from which it can be obtained. As the SPSC domain is larger than the 1-Euclidean domain, we hope that our characterization and algorithms can be useful for dealing with problems for which initial intuitions were obtained in the 1Euclidean setting, but where a combinatorial perspective is necessary; for example, in another paper we used this approach to find an improved algorithm for the egalitarian variant of the Monroe multiwinner rule (Skowron et al. 2015).

\section{Preliminaries}

Given a positive integer $s$, we write $[s]$ to denote the set $\{1, \ldots, s\}$. An election is a pair $(C, V)$, where $C=\left\{c_{1}, \ldots, c_{m}\right\}$ is a set of candidates and $V=\left(v_{1}, \ldots, v_{n}\right)$ is a list of voters. Each voter $v \in V$ is described by her preference order, or vote, $\succ_{v}$, which is a linear order over $C$. Given a voter $v \in V$ and a candidate $c \in C$, we denote by $\operatorname{pos}(v, c)$ the position of $c$ in $\succ_{v}$ : we have $\operatorname{pos}(v, c)=1$ if $c$ is $v$ 's most preferred candidate and $\operatorname{pos}(v, c)=m$ if $c$ is $v$ 's least preferred candidate. Voter $v$ 's most preferred candidate is denoted by top $(v)$. We refer to the list $\left(\succ_{v}\right)_{v \in V}$ as the preference profile. In what follows, we use the terms "election", "preferences", and "profile" interchangeably.

Given an election $E=(C, V)$ and a subset of candidates $D \subset C$, let $\left.V\right|_{D}$ denote the profile obtained by restricting the preference order of each voter in $V$ to $D$. Throughout the paper, we assume that the candidate set $C$ remains fixed, but the voter population may vary. The concatenation of two voter lists $U$ and $V$ is denoted by $U+V$; if $U$

\footnotetext{
1 The algorithm of Doignon and Falmagne (1994) was later rediscovered by Elkind and Faliszewski (2014).
} 
consists of a single vote $u$, we simply write $u+V$. We say that a list $U$ is a sublist of a list $V$ (and write $U \subseteq V$ ) if $U$ can be obtained from $V$ by deleting voters. An election $\left(C^{\prime}, V^{\prime}\right)$ is said to be a subelection of an election $(C, V)$ if $C^{\prime} \subseteq C$ and there exists a $U \subseteq V$ such that $V^{\prime}=\left.U\right|_{C^{\prime}}$

Single-crossing (also known as intermediate or order-restricted) preferences, first studied by Mirrlees (1971) and Roberts (1977), capture settings where the voters can be ordered along a single axis according to their beliefs.

Definition 1 An election $E=(C, V)$ with $C=\left\{c_{1}, \ldots, c_{m}\right\}, V=\left(v_{1}, \ldots, v_{n}\right)$ is single-crossing $(S C)$ (with respect to the given order of voters) if for every pair of candidates $a, b$ such that $a \succ_{v_{1}} b$ there exists a $t \in[n]$ such that $\left\{i \in[n] \mid a \succ_{v_{i}} b\right\}$ $=[t]$.

Intuitively, as we sweep from left to right through the list of voters in a single-crossing election, the relative order of every pair of candidates changes at most once.

We emphasize that we define single-crossing preferences with respect to a fixed order of the voters. Alternatively, one could define an election to be single-crossing if the voters can be ordered so that the condition in Definition 1 is satisfied. Computationally, these two definitions are essentially equivalent: given an election, one can efficiently check whether there exists an ordering of the voters satisfying the condition in Definition 1, and, if so, find such an ordering in polynomial time (Doignon and Falmagne 1994; Elkind et al. 2012; Bredereck et al. 2013). We state our results in terms of the former definition, as this enables us to formulate the intermediate lemmas more succinctly; however, the reader can verify that our main result (Theorem 9) remains true under the latter definition.

While single-crossing elections are defined in terms of an ordering of the voters, the definition of single-peaked elections (Black 1948) refers to an ordering of the candidates.

Definition 2 The preference order of a voter $v$ with top $(v)=c$ in an election $E$ $=(C, V)$ is single-peaked with respect to an order $\triangleleft$ over $C$ if for every pair of candidates $a, b$ such that $a \triangleleft b \triangleleft c$ or $c \triangleleft b \triangleleft a$ it holds that $c \succ_{v} b \succ_{v} a$. An election $E=(C, V)$ is single-peaked with respect to $\triangleleft$ if every vote in $V$ is single-peaked with respect to $\triangleleft$ in $E$; in this case, $\triangleleft$ is called a societal axis for $E$. $E$ is single-peaked $(S P)$ if it is single-peaked with respect to some societal axis $\triangleleft$.

There are polynomial-time algorithms that given an election $E$ decide if it is singlepeaked and, if so, compute a societal axis $\triangleleft$ such that $E$ is single-peaked with respect to $\triangleleft$ (Bartholdi and Trick 1986; Doignon and Falmagne 1994; Escoffier et al. 2008). Thus we can assume without loss of generality that when we are given a single-peaked election, we are also provided a societal axis that witnesses this.

\section{Characterization of the SPSC domain}

Fix a candidate set $C$ and consider the domain of all elections over $C$ that are singlepeaked with respect to some order of the candidates and single-crossing with respect 
to the order in which the voters are listed. We will refer to this domain as the SPSC domain; note that this domain contains elections that differ form each other in terms of the number of voters.

Our main result - the characterization of the SPSC domain - is based on the concept of minimally rich single-crossing elections. An election is minimally rich if every candidate is ranked first by at least one voter. The notion of minimal richness was used, e.g., by Cornaz et al. (2012, 2013) and Skowron et al. (2015) in the context of voting rules with computationally hard winner-determination procedures. ${ }^{2}$ Interestingly, minimal richness turns out to be closely related to the two domains restrictions that are the focus of this paper: every minimally rich single-crossing (MRSC) election is single-peaked. This observation follows from the discussion of top monotonicity by Barberà and Moreno (2011) and from a recent characterization of Puppe (2018, Corollary 3); for the sake of completeness, we provide a very simple, direct proof.

Proposition 3 A minimally rich single-crossing election is single-peaked with respect to the axis given by the preference order of the first voter.

Proof Let $E=(C, V)$ be a minimally rich single-crossing election. Without loss of generality, we assume that the preference order of the first voter in $V$ is $c_{1} \succ c_{2} \succ$ $\cdots \succ c_{m}$. We will show that each vote in $V$ is single-peaked with respect to this order. It suffices to argue that for each voter $v_{i} \in V$ and for all $1 \leq j<k<\ell \leq m$ it holds that $c_{k}$ is not ranked last among $c_{j}, c_{k}$ and $c_{\ell}$ in $v_{i}$ 's preference order.

Suppose for the sake of contradiction that there is a triple of candidates $c_{j}, c_{k}$ and $c_{\ell}, j<k<\ell$, such that some voter $v_{i}$ in $V^{\prime}$ ranks $c_{k}$ below $c_{j}$ and $c_{\ell}$. Since $(C, V)$ is minimally rich, there is another voter $v_{i^{\prime}}$ that ranks $c_{k}$ first. It must be that $i^{\prime}>i$ because $c_{j} \succ_{v_{1}} c_{k}, c_{j} \succ_{v_{i}} c_{k}, c_{k} \succ_{v_{i^{\prime}}} c_{j}$ and $(C, V)$ is single-crossing. Yet, we have $c_{k} \succ_{v_{1}} c_{\ell}, c_{\ell} \succ_{v_{i}} c_{k}$, and $c_{k} \succ_{v_{i^{\prime}}} c_{\ell}$, a contradiction.

Proposition 3 suggests that the SPSC domain is closely related to the domain of all single-crossing elections over $C$ that are minimally rich. However, it is evident that there are elections that are single-peaked and single-crossing, but not minimally rich. To see this, it suffices to note that the SPSC domain is closed under voter deletion: Given an SPSC election and some candidate $c$ in it, we can delete all voters that rank $c$ first to obtain an SPSC election that is not minimally rich. This observation motivates us to study the closure of the minimally rich single-crossing domain under voter deletion.

Definition 4 An election $E^{\prime}=\left(C, V^{\prime}\right)$ is pre-minimally rich single-crossing (pre$M R S C)$ if there exists a minimally rich single-crossing election $E=(C, V)$ such that $V^{\prime} \subseteq V$.

Clearly, pre-MRSC elections are single-crossing and single-peaked. The main result of this paper is that the converse is also true: every SPSC election is pre-MRSC. Before

\footnotetext{
2 While the authors of these papers spoke of narcissistic profiles rather than minimally rich ones, the latter term would have been more precise. Formally, narcissistic profiles, introduced by Bartholdi and Trick (1986), arise when the set of candidates coincides with the set of voters and each voter ranks him or herself first. Such profiles are, of course, minimally rich, but there are minimally rich profiles that are not narcissistic. In an early version of this paper we also used the term 'narcissistic elections' to refer to minimally rich elections, and we are grateful to the reviewers for pointing out this distinction.
} 
we give the proof of this result, in order to build up the readers' intuition, we present a general construction of single-crossing elections that are not pre-MRSC.

Example 5 Consider a minimally rich single-crossing election $E=(C, V)$ with $|C|$ $\geq 3$ and add a new candidate $x \notin C$ to the top of each vote. Let $E^{\prime}=\left(C \cup\{x\}, V^{\prime}\right)$ denote the resulting election. We claim that $E^{\prime}$ is not pre-MRSC. Indeed, suppose that there exists a minimally rich single-crossing election $E^{\prime \prime}=\left(C \cup\{x\}, V^{\prime \prime}\right)$ with $V^{\prime} \subseteq V^{\prime \prime}$. Let $a, b$, and $c$ be three distinct candidates in $C$, and for each $z \in\{a, b, c\}$ let $v_{z}$ be some vote in $V^{\prime \prime}$ that ranks $z$ first (the existence of $v_{z}$ follows from the fact that $E^{\prime \prime}$ is minimally rich). Since $E^{\prime \prime}$ is single-crossing, the voters that rank $x$ first form a contiguous block in $V^{\prime \prime}$. At least two of the voters in $\left\{v_{a}, v_{b}, v_{c}\right\}$ have to appear on the same side of this block. Assume without loss of generality that both $v_{a}$ and $v_{b}$ precede the block of voters that rank $x$ first (and hence precede all voters from $V^{\prime}$ ), and $v_{a}$ precedes $v_{b}$. Since $E$ was minimally rich, there is a vote $v_{a}^{\prime}$ in $V^{\prime}$ that ranks $a$ second (just below $x$ ). However, this is a contradiction, since $v_{a}$ and $v_{a}^{\prime}$ rank $a$ above $b, v_{b}$ ranks $b$ above $a$, and $v_{b}$ appears between $v_{a}$ and $v_{a}^{\prime}$ in $V^{\prime \prime}$.

The following two lemmas will be useful in our discussion. The first one provides a characterization of votes that can be inserted into a single-crossing election so that it remains single-crossing. The proof of this lemma follows directly from the definition of single-crossing preferences.

Lemma 6 Consider a single-crossing election $E=(C, V)$, where $C=\left\{c_{1}, \ldots, c_{m}\right\}$ and $V=\left(v_{1}, \ldots, v_{n}\right)$.

1. The election $E^{*}=\left(C, V^{*}\right)$ obtained from $E$ by inserting a vote $v^{*}$ right after a vote $v_{i}, i \in[n-1]$, is single-crossing if and only if $v^{*}$ has the following property: for every pair of candidates $c_{j}, c_{\ell} \in C$ it holds that if $c_{j} \succ_{v^{*}} c_{\ell}$ then $c_{j} \succ_{v_{i}} c_{\ell}$ or $c_{j} \succ_{v_{i+1}} c_{\ell}$.

2. The election $E^{+}=\left(C, V^{+}\right)$obtained from $E$ by inserting a vote $v^{+}$right after $v_{n}$ (i.e., $V^{+}=V+v^{+}$) is single-crossing if and only if $v^{+}$has the following property: for every pair of candidates $c_{j}, c_{\ell} \in C$ it holds that if $c_{j} \succ_{v^{+}} c_{\ell}$ then either $c_{j} \succ_{v_{n}} c_{\ell}$ or $c_{\ell} \succ_{v_{i}} c_{j}$ for all $i \in[n]$.

3. The election $E^{-}=\left(C, V^{-}\right)$obtained from $E$ by inserting a vote $v^{-}$right before $v_{1}$ (i.e., $V^{-}=v^{-}+V$ ) is single-crossing if and only if $v^{-}$has the following property: for every pair of candidates $c_{j}, c_{\ell} \in C$ it holds that if $c_{j} \succ_{v^{-}} c_{\ell}$ then either $c_{j} \succ_{v_{1}} c_{\ell}$ or $c_{\ell} \succ_{v_{i}} c_{j}$ for all $i \in[n]$.

Lemma 6 can be interpreted as a procedure for building a maximal (in terms of inclusion) single-crossing election. Indeed, for a given single-crossing election $E$ it tells us how to extend it with a single vote-not yet present in $E$-so that the election remains single-crossing, or informs us that such an extension is impossible. By applying the lemma repeatedly, we eventually obtain a single-crossing election that cannot be extended any further.

Our second lemma relates an order of the voters witnessing that the election is single-crossing and an axis witnessing that the election is single-peaked. 
Lemma 7 Suppose that election $E=(C, V)$ with $C=\left\{c_{1}, \ldots, c_{m}\right\}, V$ $=\left(v_{1}, \ldots, v_{n}\right)$ is single-crossing as well as single-peaked with respect to the candidate order $c_{1} \triangleleft \cdots \triangleleft c_{m}$. Suppose that the top-ranked candidate in $v_{1}$ is $c_{i}$ and the top-ranked candidate in $v_{n}$ is $c_{j}$ for some $i \leq j$. Then the most preferred candidate of each voter lies between $c_{i}$ and $c_{j}$, i.e., if $c_{\ell}$ is the top-ranked candidate in some $v_{k}$ in $V$, then $i \leq \ell \leq j$.

Proof Suppose that for some vote $v_{k}, k \in[n]$, the top-ranked candidate in $v_{k}$ is $c_{\ell}$ for some $\ell<i$. Since $E$ is single-peaked with respect to $\triangleleft, c_{i}$ appears above $c_{\ell}$ in $v_{n}$. Therefore, the pair of candidates $\left(c_{i}, c_{\ell}\right)$ and the triple of votes $\left(v_{1}, v_{k}, v_{n}\right)$ provide a witness that $E$ is not single-crossing.

Similarly, suppose that for some vote $v_{k}, k \in[n]$, the top-ranked candidate in $v_{k}$ is $c_{\ell}$ for some $\ell>j$. Since $E$ is single-peaked with respect to $\triangleleft, c_{j}$ appears above $c_{\ell}$ in $v_{1}$. Therefore, the pair of candidates $\left(c_{j}, c_{\ell}\right)$ and the triple of votes $\left(v_{1}, v_{k}, v_{n}\right)$ provide a witness that $E$ is not single-crossing.

Before we present our main result, we will prove one more useful lemma: we will show that we can take an SPSC election $E$ and prepend a vote that orders the candidates in the same way as some axis witnessing that $E$ is single-peaked, so that the resulting election remains single-crossing.

Given an axis $\triangleleft$ for an election $E=(C, V)$, let $v_{\triangleleft}$ be the vote that corresponds to $\triangleleft$, i.e., for every $c_{k}, c_{\ell} \in C$ it holds that $c_{k}$ is ranked above $c_{\ell}$ in $v_{\triangleleft}$ if and only if $c_{k} \triangleleft c_{\ell}$.

Lemma 8 Suppose that election $E=(C, V)$ with $C=\left\{c_{1}, \ldots, c_{m}\right\}, V$ $=\left(v_{1}, \ldots, v_{n}\right)$ is SPSC. Then there exists some axis $\triangleleft$ such that $E$ is single-peaked with respect to $\triangleleft$ and the election $\left(C, v_{\triangleleft}+V\right)$ is also SPSC.

Proof If $E$ is single-peaked with respect to some axis $\triangleleft$ then the election $\left(C, v_{\triangleleft}+V\right)$ is single-peaked. To show that $\triangleleft$ can be chosen so that $\left(C, v_{\triangleleft}+V\right)$ is single-crossing, we proceed as follows. We pick an arbitrary axis $\triangleleft$ witnessing that $E$ is single-peaked, and try to prepend it to $V$. If this leads to an election that is not single-crossing, we find a "minimal" pair of candidates that violates the single-crossing property, and modify $\triangleleft$ based on this pair. We then show that our modification is legal, i.e., it results in another axis witnessing that our election is single-peaked. Further, we show that this modification step can be executed at most $m$ times. It follows that eventually we obtain a single-crossing election.

Suppose that the top-ranked candidate in $v_{1}$ is $c_{i}$ and the top-ranked candidate in $v_{n}$ is $c_{j}$. Consider some axis $\triangleleft$ such that $E$ is single-peaked with respect to $\triangleleft$ and $c_{i} \triangleleft c_{j}$. We say that a pair of candidates $\left(c_{k}, c_{\ell}\right)$ is violating for $\triangleleft$ if $c_{k} \triangleleft c_{\ell}, c_{\ell} \succ_{v_{1}} c_{k}$, and $c_{k} \succ_{v_{n}} c_{\ell}$. By the third claim of Lemma 6, the election $\left(C, v_{\triangleleft}+V\right)$ is not singlecrossing if and only if there exists some violating pair for $\triangleleft$. Observe that if a pair $\left(c_{k}, c_{\ell}\right)$ is violating for $\triangleleft$ then $c_{k} \triangleleft c_{i}$ and $c_{j} \triangleleft c_{\ell}$. Indeed, if $c_{k}=c_{i}$ or $c_{i} \triangleleft c_{k}$, then $v_{\triangleleft}$ and $v_{1}$ agree on $\left(c_{k}, c_{\ell}\right)$, and if $c_{\ell}=c_{j}$ or $c_{\ell} \triangleleft c_{j}$ then $v_{\triangleleft}$ and $v_{n}$ disagree on $\left(c_{k}, c_{\ell}\right)$.

Given an axis $\triangleleft$ and a pair of candidates $a, b$ with $a \triangleleft b$, define:

$$
\min _{\triangleleft}\{a, b\}=a, \quad \max _{\triangleleft}\{a, b\}=b .
$$


We claim that if $\left(c_{k}, c_{\ell}\right)$ and $\left(c_{k^{\prime}}, c_{\ell^{\prime}}\right)$ are two violating pairs, then $\left(\max _{\triangleleft}\left\{c_{k}, c_{k^{\prime}}\right\}\right.$, $\left.\min _{\triangleleft}\left\{c_{\ell}, c_{\ell^{\prime}}\right\}\right)$ is a violating pair as well. Indeed, we can assume without loss of generality that $\max _{\triangleleft}\left\{c_{k}, c_{k^{\prime}}\right\}=c_{k^{\prime}}$. Then if $\min _{\triangleleft}\left\{c_{\ell}, c_{\ell^{\prime}}\right\}=c_{\ell^{\prime}}$, our claim is immediate, so it suffices to consider the case where $\min _{\triangleleft}\left\{c_{\ell}, c_{\ell^{\prime}}\right\}=c_{\ell}$, i.e.,

$$
c_{k} \triangleleft c_{k}^{\prime} \triangleleft c_{i} \triangleleft c_{j} \triangleleft c_{\ell} \triangleleft c_{\ell^{\prime}}
$$

Then $c_{\ell^{\prime}} \succ_{v_{1}} c_{k^{\prime}}$ (since $\left(c_{k^{\prime}}, c_{\ell^{\prime}}\right)$ is a violating pair) and $c_{\ell} \succ_{v_{1}} c_{\ell^{\prime}}$ (since $v_{1}$ is single-peaked with respect to $\triangleleft$ and ranks $c_{i}$ first) and hence $c_{\ell} \succ_{v_{1}} c_{k^{\prime}}$. By a similar argument, $c_{k^{\prime}} \succ_{v_{n}} c_{\ell}$. Thus, $\left(c_{k^{\prime}}, c_{\ell}\right)$ is a violating pair.

Let $\mathcal{S}_{\triangleleft}$ be the set of all violating pairs for $\triangleleft$. We say that $\left(c_{p}, c_{q}\right) \in \mathcal{S}_{\triangleleft}$ is the minimal violating pair for $\triangleleft$ if for each $\left(c_{k}, c_{\ell}\right) \in \mathcal{S}_{\triangleleft}$ it holds that (a) $c_{k} \triangleleft c_{p}$ or $c_{k}=c_{p}$, and (b) $c_{q} \triangleleft c_{\ell}$ or $c_{\ell}=c_{q}$.

The argument in the previous paragraph shows that for every axis $\triangleleft$ such that $\mathcal{S}_{\triangleleft} \neq \varnothing$ there is a unique minimal violating pair. If $\left(c_{p}, c_{q}\right)$ is the minimal violating pair for $\triangleleft$, set

$$
\delta(\triangleleft)=\left|\left\{c \mid c_{p} \triangleleft c \triangleleft c_{i}\right\}\right|
$$

Now, pick an arbitrary axis $\triangleleft$ such that $E$ is single-peaked with respect to $\triangleleft$; assume without loss of generality that $c_{1} \triangleleft \cdots \triangleleft c_{m}$. If there are no violating pairs for $\triangleleft$, we are done. Otherwise, let $\left(c_{p}, c_{q}\right)$ be the minimal violating pair for $\triangleleft$. Consider the axis $\triangleleft^{\prime}$ obtained from $\triangleleft$ by swapping the "tails" $\left(c_{1}, \ldots, c_{p}\right)$ and $\left(c_{q}, \ldots, c_{m}\right)$. Formally, $\triangleleft^{\prime}$ is given by

$$
c_{m} \triangleleft^{\prime} c_{m-1} \triangleleft^{\prime} \cdots \triangleleft^{\prime} c_{q} \triangleleft^{\prime} c_{p+1} \triangleleft^{\prime} \cdots \triangleleft^{\prime} c_{q-1} \triangleleft^{\prime} c_{p} \triangleleft^{\prime} \cdots \triangleleft^{\prime} c_{1} .
$$

We will now prove that every vote in $V$ is single-peaked with respect to $\triangleleft^{\prime}$. Indeed, suppose that this is not the case for some vote $v \in V$, and let $c_{t}$ be the top-ranked candidate in $v$. Note that by Lemma 7 we have $i \leq t \leq j$. Let $C^{--}=\left\{c_{1}, \ldots, c_{p}\right\}$, $C^{-}=\left\{c_{p+1}, \ldots, c_{t-1}\right\}, C^{+}=\left\{c_{t+1}, \ldots, c_{q-1}\right\}, C^{++}=\left\{c_{q}, \ldots, c_{m}\right\}$. We know that $v$ is single-peaked with respect to $\triangleleft$; hence, it is not single-peaked with respect to $\triangleleft^{\prime}$ if and only if (a) $a \succ_{v} b$ for some $a \in C^{--}, b \in C^{+}$or (b) $c \succ_{v} d$ for some $c \in C^{++}, d \in C^{-}$. We will now argue that neither of these cases is possible.

Consider first case (a). Since $p<i$ and $q>j, v$ 's most preferred candidate in $C^{--}$is $c_{p}$, and his least preferred candidate in $C^{+}$is $c_{q-1}$, so it has to be the case that $v$ prefers $c_{p}$ to $c_{q-1}$. On the other hand, $v_{1}$ prefers $c_{q}$ to $c_{p}$ (because $\left(c_{p}, c_{q}\right)$ is a violating pair) and $c_{q-1}$ to $c_{q}$ (because $q>i$ ), which means that $c_{q-1} \succ_{v_{1}} c_{p}$. Further, $v_{n}$ prefers $c_{q-1}$ to $c_{p}$, since otherwise $\left(c_{p}, c_{q-1}\right)$ would be a violating pair, a contradiction with $\left(c_{p}, c_{q}\right)$ being the minimal violating pair for $\triangleleft$. Thus, the pair $\left(c_{p}, c_{q-1}\right)$ and the triple $\left(v_{1}, v, v_{n}\right)$ provide a witness that $E$ is not single-crossing, a contradiction.

The argument for case (b) is similar. Since $p<i$ and $q>j$, $v$ 's most preferred candidate in $C^{++}$is $c_{q}$, and his least preferred candidate in $C^{-}$is $c_{p+1}$, so it has to be the case that $v$ prefers $c_{q}$ to $c_{p+1}$. On the other hand, $v_{n}$ prefers $c_{p}$ to $c_{q}$ ( since $\left(c_{p}, c_{q}\right)$ is a violating pair); as $p<j$, this implies that $v_{n}$ prefers $c_{p+1}$ to $c_{q}$. Further, $v_{1}$ prefers 
$c_{p+1}$ to $c_{q}$, since otherwise $\left(c_{p+1}, c_{q}\right)$ would be a violating pair, a contradiction with $\left(c_{p}, c_{q}\right)$ being the minimal violating pair for $\triangleleft$. Thus, the pair $\left(c_{p+1}, c_{q}\right)$ and the triple $\left(v_{1}, v, v_{n}\right)$ provide a witness that $E$ is not single-crossing, a contradiction.

We have shown that $E$ is single-peaked with respect to $\triangleleft^{\prime}$. We will now argue that $\delta\left(\triangleleft^{\prime}\right)>\delta(\triangleleft)$. To this end, we will show that if $\left(c_{k}, c_{\ell}\right)$ is a violating pair for $\triangleleft^{\prime}$, then $k \geq q+1$; this would imply $\delta\left(\triangleleft^{\prime}\right)=(k-q)+(i-p-1)>i-p-1=\delta(\triangleleft)$.

Note first that if $\left(c_{k}, c_{\ell}\right)$ is a violating pair for $\triangleleft^{\prime}$, then $c_{k}$ has to be located to the left of $c_{i}$ with respect to $\triangleleft^{\prime}$, so either $k \in\{m, \ldots, q\}$ or $k \in\{p+1, \ldots, i-1\}$. Similarly, $c_{\ell}$ has to be located to the right of $c_{j}$ with respect to $\triangleleft^{\prime}$, so either $\ell \in\{j+1, \ldots, q-1\}$ or $\ell \in\{p, \ldots, 1\}$.

We consider the following cases and conclude that each of them is impossible.

1. $k \in\{p+1, \ldots, i-1\}, \ell \in\{j+1, \ldots, q-1\}$. Then $\left(c_{k}, c_{\ell}\right)$ is a violating pair for $\triangleleft$, a contradiction with our choice of $\left(c_{p}, c_{q}\right)$.

2. $k \in\{p+1, \ldots, i-1\}, \ell \in\{p, \ldots, 1\}$. Since $v_{1}$ is single-peaked with respect to $\triangleleft$ and $p<i, v_{1}$ prefers $c_{k}$ to $c_{\ell}$, so $\left(c_{k}, c_{\ell}\right)$ cannot be a violating pair for $\triangleleft^{\prime}$.

3. $k=q, \ell \in\{j+1, \ldots, q-1\}$. Since $v_{n}$ is single-peaked with respect to $\triangleleft$ and $j<\ell<q, v_{n}$ prefers $c_{\ell}$ to $c_{k}$, so $\left(c_{k}, c_{\ell}\right)$ cannot be a violating pair for $\triangleleft^{\prime}$.

4. $k=q, \ell \in\{p, \ldots, 1\}$. Since $\left(c_{p}, c_{q}\right)$ is a violating pair with respect to $\triangleleft, v_{1}$ prefers $c_{q}$ to $c_{p}$. Since $v_{1}$ is single-peaked with respect to $\triangleleft$ and $\ell \leq p<i, v_{1}$ prefers $c_{p}$ to $c_{\ell}$. Hence, we have $c_{k}=c_{q} \succ_{v_{1}} c_{p} \succ_{v_{1}} c_{\ell}$, so $\left(c_{k}, c_{\ell}\right)$ cannot be a violating pair for $\triangleleft^{\prime}$.

Thus, the only remaining possibility is that $k>q$ and therefore $\delta\left(\triangleleft^{\prime}\right)>\delta(\triangleleft)$.

We now apply the same argument to $\triangleleft^{\prime}$. If $v_{\triangleleft^{\prime}}+V$ is single-crossing, we are done, and otherwise we obtain an axis $\triangleleft^{\prime \prime}$ such that $E$ is single-peaked with respect to $\triangleleft^{\prime \prime}$ and $\delta\left(\triangleleft^{\prime \prime}\right)>\delta\left(\triangleleft^{\prime}\right)$. We then continue in the same manner; since $\delta(\triangleleft) \leq m$ for every

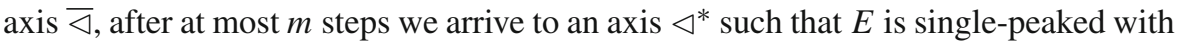
respect to $\triangleleft^{*}$ and $v_{\triangleleft^{*}}+V$ is single-crossing. This completes the proof.

We are now ready to prove our main result.

Theorem 9 An election is SPSC if and only if it is pre-MRSC.

Proof We have already argued that every pre-MRSC election is SPSC (see Proposition 3). For the converse direction, we proceed as follows.

Consider an SPSC election $E=(C, V)$ with $C=\left\{c_{1}, \ldots, c_{m}\right\}, V=\left(v_{1}, \ldots, v_{n}\right)$. By Lemma 8 we can assume that $E$ is single-peaked with respect to the candidate order $c_{1} \triangleleft \cdots \triangleleft c_{m}$, and $v_{1}$ is given by $c_{1} \succ \cdots \succ c_{m}$. We now show how to extend $E$ to a single-crossing minimally rich election.

For every $c_{i} \in C$, let $V_{i}$ be the list of voters who rank $c_{i}$ first. Consider two candidates $c_{i}, c_{j} \in C$ such that $V_{i} \neq \varnothing, V_{j} \neq \emptyset$ and $i<j$. Since $E$ is single-crossing and $c_{i} \succ_{v_{1}} c_{j}$, in $V$ all voters from $V_{i}$ appear before those from $V_{j}$.

Let $c_{s}$ be the first candidate for which $V_{s}=\emptyset$. Note that $s>1$, since $c_{1}$ is ranked first by $v_{1}$. We have $V_{r} \neq \varnothing$ for all $r<s$, and, in particular, $V_{s-1} \neq \emptyset$. Let $u$ be the last voter in $V_{s-1}$. Since $u$ 's preference order is single-peaked with respect to $\triangleleft$, his vote can be written as $c_{s-1} \succ c_{s-2} \succ \cdots \succ c_{s-\ell} \succ c_{s} \succ \cdots$ for some $\ell \geq 1$. Now consider the vote $v$ obtained by moving $c_{s}$ to the top of $u$ without changing the relative 
order of the remaining candidates. It is immediate that $v$ is single-peaked with respect to $\triangleleft$ : intuitively, when ranking candidates, $v$ starts at $c_{s}$, then moves one step to the left, then emulates $u$. We claim that the election obtained by inserting $v$ right after $u$ remains single-crossing.

Suppose that $u \neq v_{n}$, and let $w$ be the voter that appears right after $u$ in $V$. The most preferred candidate of $w$ is some $c_{q}$ for $q>s$. Since $w$ is single-peaked with respect to $\triangleleft$ and ranks $c_{q}$ first, $v$ and $w$ agree on all pairs of the form $\left(c_{s}, c_{s-r}\right), r \in[\ell]$. On the other hand, $u$ and $v$ agree on all other pairs of candidates. By the first claim of Lemma 6, we are done.

Now, suppose that $u=v_{n}$, i.e., $v$ is the last voter in the new election. The only pairs of candidates that $u$ and $v$ disagree on are $\left(c_{s}, c_{s-1}\right), \ldots,\left(c_{s}, c_{s-\ell}\right)$. On the other hand, both $v_{1}$ and $u$ (and hence all voters in $V$ ) rank $c_{s}$ below $c_{s-r}$ for all $r=1, \ldots, \ell$. By the second claim of Lemma 6, we are done.

We have successfully added a vote that ranks $c_{s}$ first. By repeating this construction for all candidates that had no first-place votes in the original election, we obtain a minimally rich profile that is single-crossing and single-peaked with respect to $\triangleleft$. This completes the proof.

Theorem 9 is constructive and, in particular, it implies a polynomial-time algorithm that, given an SPSC election $E$, finds an MRSC election that can be obtained from $E$ by adding voters. This algorithm consists of two steps: (a) finding the single-peaked axis that can be used as the first vote (or checking that the first vote already defines an axis witnessing that the election is single-peaked), and (b) adding the votes as in the proof. The second step requires time $O\left(n+m^{2}\right)$ : we have to look at the first position of each vote, and at most $m$ times we have to create a new vote in $O(m)$ time. Unfortunately, the first step may be significantly slower as Lemma 8 implies only an $O\left(n m+m^{3}\right)$ algorithm for the problem (the $O(n m)$ part stems from computing a single-peaked axis using the algorithm of Doignon and Falmagne (1994) or Escoffier et al. (2008), and the $O\left(\mathrm{~m}^{3}\right)$ part captures the complexity of finding violating pairs and manipulating the axis).

Altogether, the running time of the algorithm implied by Theorem 9 is $O\left(n m+m^{3}\right)$. The next theorem provides an alternative $O\left(\mathrm{~nm}^{2}\right)$ algorithm by looking at the problem from a very different angle.

Theorem 10 There exists an algorithm that given an election $E=(C, V)$ decides whether it is pre-MRSC, and, if so, constructs an MRSC election $E^{\prime}=\left(C, V^{\prime}\right)$ such that $V \subseteq V^{\prime}$, in time $O\left(\mathrm{~nm}^{2}\right)$.

Proof Suppose that $C=\left\{c_{1}, \ldots, c_{m}\right\}, V=\left(v_{1}, \ldots, v_{n}\right)$. Let $C^{*}$ be the set of candidates that receive no first-place votes from the voters in $V$.

Suppose there exists an MRSC election $E^{\prime}=\left(C, V^{\prime}\right)$ with $V \subseteq V^{\prime}$. We first present some observations on the structure of $E^{\prime}$. We can assume without loss of generality that for each $c_{j} \in C^{*}$ there is a unique vote $v_{j}^{*}$ in $V^{\prime}$ that ranks $c_{j}$ first. Consider an arbitrary candidate $c \in C \backslash\left\{c_{j}\right\}$. Suppose first that $c \succ_{v_{1}} c_{j}, c_{j} \succ_{v_{n}} c$. Then there is a unique index $i \in[n-1]$ such that $c \succ_{v_{i}} c_{j}, c_{j} \succ_{v_{i+1}} c$. Since $E^{\prime}$ is single-crossing, it has to be the case that $v_{j}^{*}$ appears after $v_{i}$ in $V^{\prime}$. Similarly, if $c_{j} \succ_{v_{1}} c, c \succ_{v_{n}} c_{j}$, then there is a unique index $i \in[n-1]$ such that $c_{j} \succ_{v_{i}} c, c \succ_{v_{i+1}} c_{j}$, and $v_{j}^{*}$ appears 
before $v_{i+1}$ in $V^{\prime}$. Finally, if all voters in $V$ prefer $c$ to $c_{j}$, then in $V^{\prime}$ voter $v_{j}^{*}$ appears either before $v_{1}$ or after $v_{n}$.

We can now present the high-level idea behind our algorithm. For each candidate $c_{j} \in C^{*}$ our algorithm determines all the constraints on the position of $v_{j}^{*}$, as described above. If for some candidate the constraints are incompatible with each other, it stops and outputs "no". Otherwise, it outputs "yes"; we will show how to construct a minimally rich single-crossing election $E=\left(C, V^{\prime}\right)$ with $V \subseteq V^{\prime}$ in this case.

In more detail, our algorithm first checks whether $E$ is single-crossing and outputs "no" if this is not the case. Thus, from now on we will assume that $E$ is single-crossing with respect to $\left(v_{1}, \ldots, v_{n}\right)$. Then, for each $c_{j} \in C^{*}$, we construct a set $P_{j}$ as follows. Initially we set $P_{j}=\{0,1, \ldots, n\}$. We then consider all candidates in $C \backslash\left\{c_{j}\right\}$ one by one. For each $c \in C \backslash\left\{c_{j}\right\}$ we have one of the following cases:

(1) There exists an $i \in[n-1]$ such that $c \succ_{v_{i}} c_{j}, c_{j} \succ_{v_{i+1}} c$. In this case we set $P_{j}:=P_{j} \backslash\{0, \ldots, i-1\}$.

(2) There exists an $i \in[n-1]$ such that $c_{j} \succ_{v_{i}} c, c \succ_{v_{i+1}} c_{j}$. In this case we set $P_{j}:=P_{j} \backslash\{i+1, \ldots, n\}$.

(3) For all $i \in[n]$ it holds that $c \succ_{v_{i}} c_{j}$. In this case we set $P_{j}:=P_{j} \backslash\{1, \ldots, n-1\}$.

(4) For all $i \in[n]$ it holds that $c_{j} \succ_{v_{i}} c$. In this case $P_{j}$ remains unchanged.

We compute the sets $P_{j}$ for all candidates $c_{j} \in C^{*}$. If $P_{j}=\emptyset$ for some $c_{j} \in C^{*}$, we output "no"; otherwise we output "yes". We claim that this algorithm correctly decides whether $E$ is pre-MRSC.

Suppose first that the algorithm outputs "no". Then either $E$ is not single-crossing, or there exists a candidate $j \in C^{*}$ with $P_{j}=\emptyset$. In the former case the algorithm is obviously correct. Now consider the latter case. As argued above, the set $P_{j}$ encodes the possible positions with respect to $V$ for a voter that ranks $c_{j}$ first. That is, we remove an element $i, i \in[n]$, from $P_{j}$ if and only if placing a voter who ranks $c_{j}$ first after $v_{i}$ would result in an election that is not single-crossing. Similarly, we remove 0 from $P_{j}$ if and only if placing a voter who ranks $c_{j}$ first before all voters in $V$ would result in an election that is not single-crossing. Thus, if all positions have been removed from $P_{j}$, this means that it is impossible to place a voter who ranks $c_{j}$ first without turning $E$ into an election that is not single-crossing. This implies that $E$ is not pre-MRSC.

On the other hand, suppose that the algorithm outputs "yes", i.e., $P_{j} \neq \varnothing$ for all $c_{j} \in C^{*}$. Observe that for each $c_{j} \in C^{*}$ we have either $\left|P_{j}\right|=1$ or $P_{j}=\{0, n\}$. Indeed, suppose that $i, \ell \in P_{j}$ for some $c_{j} \in C^{*}$, and $i<\ell$. Voter $v_{\ell}$ ranks some candidate $c, c \neq c_{j}$, first. Now, when constructing the set $P_{j}$, our algorithm has considered $c$. If $c_{j} \succ_{v_{1}} c$, the algorithm should have removed $\ell$ from $P_{j}$. Similarly, if $c_{j} \succ_{v_{n}} c$, the algorithm should have removed $i$ from $P_{j}$. Since both $i$ and $\ell$ are still in $P_{j}$, it has to be the case that both $v_{1}$ and $v_{n}$ (and hence all voters in $V$ ) prefer $c$ to $c_{j}$. But then the algorithm has removed all elements other than 0 and $n$ from $P_{j}$ when considering $c$, so we have $i=0, \ell=n$.

Now, let $C_{i}=\left\{c_{j} \in C^{*} \mid i \in P_{j}\right\}$ for all $i \in[n]$. Consider an $i \in[n]$ such that $C_{i} \neq \emptyset$, and assume that $C_{i}=\left\{c_{j_{1}}, \ldots, c_{j_{t}}\right\}$, where $v_{i}$ ranks the candidates in $C_{i}$ as $c_{j_{1}} \succ \cdots \succ c_{j_{t}}$. Let $v_{j_{1}}^{*}$ be the vote obtained from $v_{i}$ by moving candidate $c_{j_{1}}$ to the top of $v_{i}$ without changing the relative order of the other candidates. Then, for $\ell=2, \ldots, t$ 
let $v_{j_{\ell}}^{*}$ be the vote obtained from $v_{j_{\ell-1}}^{*}$ by moving candidate $c_{j_{\ell}}$ to the top of $v_{j_{\ell-1}}^{*}$ without changing the relative order of the other candidates. Let $V_{i}^{*}=\left(v_{j_{1}}^{*}, \ldots, v_{j_{t}}^{*}\right)$ and insert the list $V_{i}^{*}$ after $v_{i}$. Further, let $C_{0}=\left\{c_{j} \in C^{*} \mid P_{j}=\{0\}\right\}$. Suppose that $C_{0}=\left\{c_{\ell_{1}}, \ldots, c_{\ell_{s}}\right\}$, where $v_{1}$ ranks the candidates in $C_{0}$ as $c_{\ell_{1}} \succ \cdots \succ c_{\ell_{s}}$. Let $v_{\ell_{1}}^{*}$ be the vote obtained from $v_{1}$ by moving candidate $c_{\ell_{1}}$ to the top of $v_{1}$ without changing the relative order of the other candidates. Then for $j=2, \ldots, s$ let $v_{\ell_{j}}^{*}$ be the vote obtained from $v_{\ell_{j-1}}^{*}$ by moving candidate $c_{\ell_{j}}$ to the top of $v_{\ell_{j-1}}^{*}$ without changing the relative order of the other candidates. Let $V_{0}^{*}=\left(v_{\ell_{s}}^{*}, \ldots, v_{\ell_{1}}^{*}\right)$, and insert the list $V_{0}^{*}$ before $v_{1}$. Let $E^{\prime}=\left(C, V^{\prime}\right)$ denote the resulting election.

Election $E^{\prime}$ is minimally rich by construction (and the assumption that our algorithm returned "yes"). To show that it is single-crossing, we apply Lemma 6 repeatedly. Specifically, consider the set $C_{i}=\left\{c_{j_{1}}, \ldots, c_{j_{t}}\right\}$ for some $i \in[n-1]$. Observe that if $P_{j}=\{i\}$ for some $j \in C^{*}$, then for all $c$ such that $c \succ_{v_{i}} c_{j}$ we have $c_{j} \succ_{v_{i+1}} c$, and for all $c$ such that $c \succ_{v_{i+1}} c_{j}$ we have $c_{j} \succ_{v_{i}} c$. Indeed, if $c \succ_{v_{i}} c_{j}$ and $c \succ_{v_{i+1}} c_{j}$, we would have removed $i$ from $P_{j}$ when considering $c$. Now, consider the vote $v_{j_{1}}^{*}$. It agrees with $v_{i}$ on all pairs of candidates not involving $c_{j_{1}}$, as well as on all pairs of the form $\left(c_{j_{1}}, c\right)$, where $c_{j_{1}} \succ_{v_{i}} c$. On the other hand, we have argued that if $c \succ_{v_{i}} c_{j_{1}}$ then $c_{j_{1}} \succ_{v_{i+1}} c$. Thus, on all pairs of candidates $v_{j_{1}}^{*}$ agrees with $v_{i}$ or $v_{i+1}$, so after we insert $v_{j_{1}}^{*}$ after $v_{i}$, the election remains single-crossing by the first claim of Lemma 6 .

Further, suppose we have already inserted $v_{j_{1}}^{*}, \ldots, v_{j_{\ell}}^{*}$ for some $\ell<t$, and we are trying to insert $v_{j_{\ell+1}}^{*}$. The only pairs of candidates on which $v_{j_{\ell+1}}^{*}$ and $v_{j_{\ell}}^{*}$ disagree are pairs of the form $\left(c_{j_{\ell+1}}, c\right)$, where $c$ is ranked above $c_{j_{\ell+1}}$ in $v_{j_{\ell}}^{*}$. Note that $v_{j \ell}^{*}$ prefers $c$ to $c_{j_{\ell+1}}$ if and only if $v_{i}$ prefers $c$ to $c_{j_{\ell+1}}$, which means that $v_{i+1}$ prefers $c_{j_{\ell+1}}$ to $c$. Thus, by the first claim of Lemma 6 the election remains single-crossing after we insert $v_{j_{\ell+1}}^{*}$. This inductive argument shows that the election remains single-crossing after we insert all votes in $V_{i}^{*}$. We can apply this argument to all sets $C_{i}, i \in[n-1]$, one by one.

Now, consider the set $C_{n}$. Observe that if $n \in P_{j}$ for some $j \in C^{*}$, then for all $c$ such that $c \succ_{v_{n}} c_{j}$ we have $c \succ_{v_{i}} c_{j}$ for all $i \in[n]$. Indeed, if $c_{j} \succ_{v_{i}} c$ for some $i \in[n-1]$, we would have removed $n$ from $P_{j}$ when considering $c$. Thus, we can apply an inductive argument similar to the one used for $C_{i}, i \in[n-1]$; the only difference is that we invoke the second claim of Lemma 6 rather than its first claim. The set $C_{0}$ can be handled similarly, using the third claim of Lemma 6 .

It remains to analyze the running time of our algorithm. Bredereck et al. (2013) describe an $O\left(\mathrm{~nm}^{2}\right)$ algorithm for checking whether a given election is single-crossing. For each $c_{j} \in C^{*}$, the set $P_{j}$ can be computed in time $O(\mathrm{~nm})$; the easiest way to achieve this is to preprocess the votes so as to represent each vote as an $m$-by- $m$ comparison matrix, which can be done in time $O\left(\mathrm{~nm}^{2}\right)$. Each of the sets $C_{i}, i \in[n] \cup\{0\}$, can be computed in time $O(m)$, and the respective vote list $V_{i}^{*}$ can be computed in time $O\left(m\left|C_{i}\right|\right)$. To establish the bound on the running time, it remains to observe that $\left|C_{0}\right|+\cdots+\left|C_{n}\right| \leq m$.

We note that we need not assume that the input election is single-crossing in the given order: given an election, we can decide whether the votes can be reordered so that it becomes single-crossing in the given order in time $O\left(\mathrm{~nm}^{2}\right)$ (Bredereck et al. 2013). 
Hence, performing this check before executing the algorithm presented in Theorem 10 does not affect the overall running time.

We finish our discussion by pointing out an interesting property of SPSC elections: If $E$ is an SPSC election then for each candidate $c$, as we sweep through the voters in the single-crossing order, the position of $c$ first rises and then falls; this property can be quite useful when deriving algorithms for SPSC elections and was used, e.g., by Skowron et al. (2015).

Proposition 11 For every election $E=(C, V)$ with $C=\left\{c_{1}, \ldots, c_{m}\right\}, V$ $=\left(v_{1}, \ldots, v_{n}\right)$ that is SPSC with respect to the voter order $\left(v_{1}, \ldots, v_{n}\right)$ and for every candidate $c \in C$ there exists a voter $v_{\ell} \in V$ such that for every pair of voters $v_{i}, v_{j}$ satisfying $j<i \leq \ell$ or $\ell \leq i<j$ it holds that $\operatorname{pos}\left(v_{j}, c\right) \geq \operatorname{pos}\left(v_{i}, c\right)$.

Proof If an election $E$ has the property described in the proposition statement, then every election obtained from $E$ by deleting voters also has this property. Thus, it suffices to give a proof for the case where $E$ is single-crossing and minimally rich. Fix a candidate $c \in C$ and let $v_{\ell}$ be some voter that ranks $c$ first. Consider two voters, $v_{j}$ and $v_{i}$, such that $j<i \leq \ell$. If $\operatorname{pos}\left(v_{j}, c\right)<\operatorname{pos}\left(v_{i}, c\right)$, there exists a candidate $c^{\prime}$ such that $v_{j}$ prefers $c$ to $c^{\prime}$, but $v_{i}$ prefers $c^{\prime}$ to $c$. However, $v_{\ell}$ ranks $c$ first, so she also prefers $c$ to $c^{\prime}$, and this is a contradiction with the assumption that $E$ is single-crossing. The case $\ell \leq i<j$ is similar.

\section{Conclusions}

We have explored the domain of all elections that are simultaneously single-peaked and single-crossing. We established a connection between minimally rich elections, single-crossing elections, and single-peaked elections that led to a characterization of the SPSC domain. Further, we have proposed two algorithms for reconstructing the minimally rich single-crossing election from which they can be obtained. Finding such an embedding may be useful for algorithms that exploit the properties of the SPSC domain, just like finding a single-peaked axis or the single-crossing order of voters is useful for some of the algorithms for single-peaked or single-crossing elections.

Acknowledgements Part of this work was done while the first author was employed by Nanyang Technological University, Singapore, and supported by National Research Foundation (Singapore) grant NRF2009-08; the first author was also supported by the European Research Council (ERC) under Grant Number 639945 (ACCORD). The second author was supported by NCN Grants 2012/06/M/ST1/00358 and 2011/03/B/ST6/01393, and by AGH University Grant 11.11.230.015. The third author was supported by Polish National Science Center grant Preludium UMO-2013/09/N/ST6/03661 and by the Foundation for Polish Science within the Homing programme (Project title: "Normative Comparison of Multiwinner Election Rules"). The authors would like to thank the anonymous AAAI and Social Choice and Welfare reviewers for their very useful feedback.

Open Access This article is distributed under the terms of the Creative Commons Attribution 4.0 International License (http://creativecommons.org/licenses/by/4.0/), which permits unrestricted use, distribution, and reproduction in any medium, provided you give appropriate credit to the original author(s) and the source, provide a link to the Creative Commons license, and indicate if changes were made. 


\section{References}

Arrow K (1951) Social choice and individual values. Wiley, Amsterdam

Ballester M, Haeringer G (2011) A characterization of the single-peaked domain. Soc Choice Welf 36(2):305-322

Barberà S, Moreno B (2011) Top monotonicity: a common root for single peakedness, single crossing and the median voter result. Games Econ Behav 73(2):345-359

Bartholdi J III, Trick M (1986) Stable matching with preferences derived from a psychological model. Oper Res Lett 5(4):165-169

Betzler N, Slinko A, Uhlmann J (2013) On the computation of fully proportional representation. J Artif Intell Res 47:475-519

Black D (1948) On the rationale of group decision-making. J Polit Econ 56(1):23-34

Brandt F, Brill M, Hemaspaandra E, Hemaspaandra LA (2015) Bypassing combinatorial protections: polynomial-time algorithms for single-peaked electorates. J Artif Intell Res 53:439-496

Bredereck R, Chen J, Woeginger G (2013) A characterization of the single-crossing domain. Soc Choice Welf 41(4):989-998

Chen J, Pruhs K, Woeginger GJ (2017) The one-dimensional Euclidean domain: finitely many obstructions are not enough. Soc Choice Welf 48(2):409-432

Conitzer V (2009) Eliciting single-peaked preferences using comparison queries. J Artif Intell Res 35:161191

Coombs C (1950) Psychological scaling without a unit of measurement. Psychol Rev 57(3):145-158

Cornaz D, Galand L, Spanjaard O (2012) Bounded single-peaked width and proportional representation. In: Proceedings of the 20th European conference on artificial intelligence, pp 270-275

Cornaz D, Galand L, Spanjaard O (2013) Kemeny elections with bounded single-peaked or single-crossing width. In: Proceedings of the 23rd international joint conference on artificial intelligence, pp 76-82

Demange G (1994) Intermediate preferences and stable coalition structures. J Math Econ 23(1):45-58

Dey P, Misra N (2016) Preference elicitation for single crossing domain. In: Proceedings of the 25th international joint conference on artificial intelligence, pp 222-228

Doignon J-P, Falmagne J-C (1994) A polynomial time algorithm for unidimensional unfolding representations. J Algorithm 16(2):218-233

Elkind E, Faliszewski P (2014) Recognizing 1-Euclidean preferences: an alternative approach. In: Proceedings of the 7th international symposium on algorithmic game theory, pp 146-157

Elkind E, Faliszewski P, Slinko A (2012) Clone structures in voters' preferences. In: Proceedings of the 13th ACM conference on electronic commerce, pp 496-513

Elkind E, Lackner M, Peters D (2017) Structured preferences. In: Endriss U (ed), Trends in computational social choice, chapter 10, pp 187-207. AI Access

Escoffier B, Lang J, Öztürk M (2008) Single-peaked consistency and its complexity. In: Proceedings of the 18th European conference on artificial intelligence, pp 366-370

Faliszewski P, Hemaspaandra E, Hemaspaandra L (2011a) The complexity of manipulative attacks in nearly single-peaked electorates. In: Proceedings of the 13th conference on theoretical aspects of rationality and knowledge, pp 228-237

Faliszewski P, Hemaspaandra E, Hemaspaandra L, Rothe J (2011b) The shield that never was: societies with single-peaked preferences are more open to manipulation and control. Inf Comput 209(2):89-107

Grandmont J (1978) Intermediate preferences and the majority rule. Econometrica 46(2):317-330

Inada K (1969) The simple majority decision rule. Econometrica 37(3):490-506

Jaeckle F, Peters D, Elkind E (2018) On recognising nearly single-crossing preferences. In: Proceedings of the 32nd AAAI conference on artificial intelligence, pp 1079-1086

Knoblauch V (2010) Recognizing one-dimensional Euclidean preference profiles. J Math Econ 46(1):1-5

Lakhani F, Peters D, Elkind E (2019) Correlating preferences and attributes: nearly single-crossing profiles. In: Proceedings of the 28th international joint conference on artificial intelligence

Meltzer AH, Richard SF (1981) A rational theory of the size of government. J Polit Econ 89(5):914-927

Mirrlees J (1971) An exploration in the theory of optimal income taxation. Rev Econ Stud 38:175-208

Misra N, Sonar C, Vaidyanathan PR (2017) On the complexity of Chamberlin-Courant on almost structured profiles. In: Proceedings of the 5th international conference on algorithmic decision theory, pp 124-138

Moulin H (1980) On strategy-proofness and single peakedness. Public Choice 35(4):437-455

Puppe C (2018) The single-peaked domain revisited: a simple global characterization. J Econ Theory 176:55-80 
Roberts KWS (1977) Voting over income tax schedules. J Public Econ 8(3):329-340

Saporiti A (2009) Strategy-proofness and single-crossing. Theor Econ 4(2):127-163

Saporiti A, Tohmé F (2006) Single-crossing, strategic voting and the median choice rule. Soc Choice Welf 26(2):363-383

Skowron P, Yu L, Faliszewski P, Elkind E (2015) The complexity of fully proportional representation for single-crossing electorates. Theor Comput Sci 569:43-57

Walsh T (2007) Uncertainty in preference elicitation and aggregation. In: Proceedings of the 22nd national conference on artificial intelligence, pp 3-8

Publisher's Note Springer Nature remains neutral with regard to jurisdictional claims in published maps and institutional affiliations. 